\title{
Bound States of the Klein-Gordon for Exponential-Type Potentials in D-Dimensions
}

\author{
Sameer M. Ikhdair \\ Physics Department, Near East University, Nicosia, North Cyprus, Turkey \\ E-mail: sikhdair@neu.edu.tr \\ Received May 27, 2011; revised June 9, 2011; accepted June 22, 2011
}

\begin{abstract}
The approximate analytic bound state solutions of the Klein-Gordon equation with equal scalar and vector exponential-type potentials including the centrifugal potential term are obtained for any arbitrary orbital quantum number $l$ and dimensional space $D$. The relativistic/non-relativistic energy spectrum formula and the corresponding un-normalized radial wave functions, expressed in terms of the Jacobi polynomials $P_{n}^{(\alpha, \beta)}(z), \alpha>-1, \beta>-1$ and $z \in[-1,+1]$ or the generalized hypergeometric functions ${ }_{2} F_{1}(a, b ; c ; z)$ have been obtained. A short-cut of the Nikiforov-Uvarov (NU) method is used in the solution. A unified treatment of the Eckart, Rosen-Morse, Hulthén and Woods-Saxon potential models can be easily derived from our general solution. The present calculations are found to be identical with those ones appearing in the literature. Further, based on the PT-symmetry, the bound state solutions of the trigonometric Rosen-Morse potential can be easily obtained.
\end{abstract}

Keywords: Approximation Scheme, Eckart-Type Potentials, Rosen-Morse-Type Potentials, Trigonometric Rosen-Morse Potential, Hulthén Potential and Woods-Saxon Potential, Klein-Gordon Equation, NU Method

\section{Introduction}

The exact solutions of the wave equations (non-relativistic or relativistic) are very important since they contain all the necessary information regarding the quantum system under consideration. However, analytical solutions are possible only in a few simple cases such as the hydrogen atom and the harmonic oscillator [1,2]. Most quantum systems could be solved only by using approximation schemes like rotating Morse potential via Pekeris approximation [3-5] and the generalized Morse potential by means of an improved approximation scheme [6]. Recently, the study of exponential-type potentials has attracted much attention from many authors (for example, cf, [7-39]). These physical potentials include the Woods- Saxon $[7,8]$, Hulthén [9-22], modified hyperbolic-type [23], ManningRosen [24-31], the Eckart [32-37], the Pöschl-Teller [38] and the Rosen-Morse [39,40] potentials.

The spherically symmetric Eckart-type potential model [41] is a molecular potential model which has been widely applied in physics [42] and chemical physics $[43,44]$ and is generally expressed as

$$
\begin{aligned}
& V(r, q)=V_{1} \operatorname{cosech} h_{q}^{2}(\alpha r)-V_{2} \operatorname{coth}_{q}(\alpha r), \\
& V_{1}, \quad V_{2}>0,-1 \leq q<0 \text { or } q>0
\end{aligned}
$$

where the coupling parameters $V_{1}$ and $V_{2}$ describe the depth of the potential well, while the screening parameter $\alpha$ is related to the range of the potential. It is a special case of the five-parameter exponential-type potential model $[45,46]$. The range of parameter $q$ was taken as $q>0$ in [47] and has been extended to $-1 \leq q<0$ or $q>0$ or even complex in [46]. The deformed hyperbolic functions given in (1) have been introduced for the first time by Arai [48] for real $q$ values. When $q$ is complex, the functions in (1) are called the generalized deformed hyperbolic functions. The Eckart-type potentials (1) can also be written in the exponential form as

$$
V(r, q)=4 V_{1} \frac{\mathrm{e}^{-2 \alpha r}}{\left(1-q \mathrm{e}^{-2 \alpha r}\right)^{2}}-V_{2} \frac{1+q \mathrm{e}^{-2 \alpha r}}{1-q \mathrm{e}^{-2 \alpha r}}
$$

The study of both bound and scattering states for the Eckart-type potential has raised a great deal of interest in the non-relativistic as well as in relativistic quantum 
mechanics. The s-wave $(l=0)$ bound-state solution of the Schrödinger equation for the Eckart potential has been widely investigated by using various methods, such as the supersymmetric (SUSY) shape invariance technology [49], point canonical transformation (PCT) method [50] and SUSY Wentzel-Kramers-Brillouin (WKB) approximation approach [51]. The bound state solutions of the s-wave Klein-Gordon (KG) equation with equally mixed Rosen-Morse-type (Eckart and Rosen--Morse well) potentials have been studied [52]. The bound state solutions of the s-wave Dirac equation with equal vector and scalar Eckart-type potentials in terms of the basic concepts of the shape-invariance approach in the SUSYQM have also been studied [34-37]. The spin symmetry and pseudospin symmetry in the relativistic Eckart potential have been investigated by solving the Dirac equation for mixed potentials [38]. Unfortunately, the wave equations for the Eckart-type potential can only be solved analytically for zero angular momentum states because of the centrifugal potential term. Some authors [32-38] studied the analytical approximations to the bound state solutions of the Schrödinger equation with Eckart potential by using the usual existing approximation scheme proposed by Greene and Aldrich [53] for the centrifugal potential term. This approximation has also been used to study analytically the arbitrary $l$-wave scattering state solutions of the Schrödinger equation for the Eckart potential $[54,55]$. The same approximation scheme for the spinorbit coupling term has been used to study the spin symmetry and pseudospin symmetry analytical solutions of the Dirac equation with the Eckart potential using the AIM [56]. Furthermore, the pseudospin symmetry analytical solutions of the Dirac equation for the Eckart potential have been found by using the SUSY WKB formalism [57]. Recently, for the first time, the approximation scheme for the centrifugal potential term has also been used in [58] to obtain the approximate analytical solution of the $\mathrm{KG}$ equation for equal scalar and vector Eckart potentials for arbitrary $l$-states by means of the functional analysis method.

This approximation for the centrifugal potential term $[9,19,53]$ has also been used to solve the Schrödinger equation [9,19], KG [10-12,20-22] and Dirac equation [20-22] for the Hulthén potential. Recently, the KG and Dirac equations have been solved in the presence of the Hulthén potential, where the energy spectrum and the scattering wave functions were obtained for spin- 0 and spin-(1/2) particles, using a more general approximation scheme for the centrifugal potential [20-22]. They found that the good approximation, however, occurs when the screening parameter $\alpha$ and the dimensionless parameter $\gamma$ are taken as $\alpha=0.1$ and $\gamma=1$, respectively, which is simply the case of the usual approxima- tion $[9,19]$. Also, other authors have recently proposed an alternative approximation scheme for the centrifugal potential to solve the Schrödinger equation for the Hulthén potential [59]. Taking $\omega=1$, their approximation can be reduced to the usual approximation $[9,19]$. Quite recently, we have also proposed a new approximation scheme for the centrifugal term [13,14].

The Nikiforov-Uvarov (NU) method [60] and other methods have also been used to solve the $D$-dimensional Schrödinger equation [61] and relativistic $D$-dimensional KG equation [62], Dirac equation $[6,15,39,40,63]$ and spinless Salpeter equation [64].

Our aim is to employ the usual approximation scheme $[53,58]$ in order to solve the $D$-dimensional radial $\mathrm{KG}$ equation for any orbital angular momentum number $l$ for the scalar and vector Eckart-type potentials using a general mathematical model of the NU method. This offers a simple, accurate and efficient scheme for the exponential-type potential models in quantum mechanics. We consider the following relationship between the scalar and vector potentials: $V(r)=V_{0}+\beta S(r)$, where $V_{0}$ and $\beta$ are arbitrary constants [51]. Under the restriction of equally mixed potentials $S(r)=V(r)$, the KG equation turns into a Schrödinger-like equation and thus the bound state solutions are very easily obtained through the well-known methods developed in the non-relativistic quantum mechanics. It is interesting to note that, this restriction include the case where $V(r)=0$ when both constants vanish, the situation where the potentials are equal $\left(V_{0}=0 ; \beta=1\right)$ and also the case where the potentials are proportional [66] when $V_{0}=0$ and $\beta= \pm 1$, which provide the equally-mixed scalar and vector potential case $V(r)= \pm S(r)$. Further, we have obtained an approximate analytic solution of the KG equation in the presence of equal scalar and vector generalized deformed hyperbolic potential functions by means of parameteric generalization of the NU method. Furthermore, for the equally-mixed scalar and vector potential case $V(r)= \pm S(r)$, we have obtained the approximate bound state rotational-vibrational (ro-vibrational) energy levels and the corresponding normalized wave functions expressed in terms of the Jacobi polynomial $P_{n}^{(\mu, v)}(x)$, where $\mu>-1, \quad v>-1$ and $x \in[-1,+1]$ for a spinzero particle in a closed form [67].

The paper is structured as follows. In Section 2, we derive a general model of the NU method valid for any central or non-central potential. In Section 3, the approximate analytical solutions of the $D$-dimensional radial KG equation with arbitrary $l$-states for equallymixed scalar and vector Eckart-type potentials and other typical potentials are obtained by means of the NU method. Also, the exact s-wave KG equation has also been solved for the Rosen-Morse-type potentials and 
other typical potentials. The relative convenience of the Eckart-type potential (Rosen-Morse-type potential) with the Hulthén potential (Woods-Saxon potential) has been studied, respectively. We make some remarks on the energy equations and the corresponding wave functions for the Eckart and Rosen-Morse well potentials in various dimensions and their non-relativistic limits in Section 4. Section 5 contains the conclusions and the outlook.

\section{Method of Analysis}

The method of analysis is briefly outlined here and the details can be found in [60]. This method was proposed to solve the second-order differential wave equation of the hypergeometric-type:

$$
\sigma^{2}(z) \psi_{n}^{\prime \prime}(z)+\sigma(z) \tilde{\tau}(z) \psi_{n}^{\prime}(z)+\tilde{\sigma}(z) \psi_{n}(z)=0
$$

where $\sigma(z)$ and $\tilde{\sigma}(z)$ are at most second-degree polynomials and $\tilde{\tau}(z)$ is a first-degree polynomial. The prime denotes the differentiation with respect to $z$. In finding a particular solution to (3), one needs to decompose the wave function $\psi_{n}(z)$ as

$$
\psi_{n}(z)=\phi_{n}(z) y_{n}(z)
$$

yielding the following hypergeometric type equation

$$
\sigma(z) y_{n}^{\prime \prime}(z)+\tau(z) y_{n}^{\prime}(z)+\lambda y_{n}(z)=0
$$

where

$$
\lambda=k+\pi^{\prime}(z)
$$

and $y_{n}(z)$ satisfying the Rodrigues relation

$$
y_{n}(z)=\frac{A_{n}}{\rho(z)} \frac{\mathrm{d}^{n}}{\mathrm{~d} z^{n}}\left[\sigma^{n}(z) \rho(z)\right]
$$

In the above equation, $A_{n}$ is a constant related to the normalization and $\rho(z)$ is the weight function satisfying the condition

with

$$
\sigma(z) \rho^{\prime}(z)+\left[\sigma^{\prime}(z)-\tau(z)\right] \rho(z)=0
$$

$$
\tau(z)=\tilde{\tau}(z)+2 \pi(z), \tau^{\prime}(z)<0
$$

Since $\rho(z)>0$ and $\sigma(z)>0$, the derivative of $\tau(z)$ should be negative [60] which is the essential condition for a proper choice of solution. The other part of the wave function in (4) can be defined as

$$
\sigma(z) \phi^{\prime}(z)-\pi(z) \phi(z)=0
$$

where

$$
\pi(z)=\frac{1}{2}\left[\sigma^{\prime}(z)-\tilde{\tau}(z)\right]
$$

*The shortcut is simple and straightforward procedure helping to avoid the difficulty in choosing the physical polynomial $\pi(z)$ and the root $k$.

$$
\pm \frac{1}{2} \sqrt{\left[\sigma^{\prime}(z)-\tilde{\tau}(z)\right]^{2}-4 \tilde{\sigma}(z)+4 k \sigma(z)}
$$

The determination of the root $k$ is the essential point in the calculation of $\pi(z)$, for which the discriminator of the square root in the last equation is being set to zero. The results in the polynomial $\pi(z)$ which is dependent on the transformation function $z(r)$. Also, the parameter $\lambda$ defined in (6) takes the following form

$$
\lambda=\lambda_{n}=-n \tau^{\prime}(z)-\frac{1}{2} n(n-1) \sigma^{\prime \prime}(z), n=0,1,2, \cdots
$$

We may construct a general recipe of the NU method valid for any central and non-central potential model. This can be achieved by comparing the following hypergeometric equation

$$
\begin{aligned}
& {\left[z\left(1-c_{3} z\right)\right]^{2} \psi_{n}^{\prime \prime}(z)+\left[z\left(1-c_{3} z\right)\left(c_{1}-c_{2} z\right)\right] \psi_{n}^{\prime}(z)} \\
& +\left(-A z^{2}+B z-C\right) \psi_{n}(z)=0
\end{aligned}
$$

with its counterpart (3) to obtain [67]

$$
\tilde{\tau}(z)=c_{1}-c_{2} z, \sigma(z)=z\left(1-c_{3} z\right), \tilde{\sigma}(z)=-A z^{2}+B z-C
$$

Further, substituting (14) into (11) gives

$$
\pi(z)=c_{4}+c_{5} z \pm\left[\left(c_{6}-c_{3} k_{+,-}\right) z^{2}+\left(c_{7}+k_{+,-}\right) z+c_{8}\right]^{1 / 2}
$$

with parametric constants

$$
\begin{aligned}
& c_{4}=\frac{1}{2}\left(1-c_{1}\right), c_{5}=\frac{1}{2}\left(c_{2}-2 c_{3}\right), c_{6}=c_{5}^{2}+A \\
& c_{7}=2 c_{4} c_{5}-B, c_{8}=c_{4}^{2}+C
\end{aligned}
$$

The discriminant under the square root sign must be set to zero and the resulting equation must be solved for $k$, it yields

$$
k_{+,-}=-\left(c_{7}+2 c_{3} c_{8}\right) \pm 2 \sqrt{c_{8} c_{9}}
$$

where

$$
c_{9}=c_{3}\left(c_{7}+c_{3} c_{8}\right)+c_{6}
$$

Inserting (17) into (15) and solving the resulting equation, we make the following choice of parameters:

$$
\begin{aligned}
& \pi(z)=c_{4}+c_{5} z-\left[\left(\sqrt{c_{9}}+c_{3} \sqrt{c_{8}}\right) z-\sqrt{c_{8}}\right] \\
& k_{-}=-\left(c_{7}+2 c_{3} c_{8}\right)-2 \sqrt{c_{8} c_{9}}
\end{aligned}
$$

Equation (9) gives

$$
\tau(z)=1-\left(c_{2}-2 c_{5}\right) z-2\left[\left(\sqrt{c_{9}}+c_{3} \sqrt{c_{8}}\right) z-\sqrt{c_{8}}\right]
$$

whose derivative must be negative:

$$
\tau^{\prime}(z)=-2 c_{3}-2\left(\sqrt{c_{9}}+c_{3} \sqrt{c_{8}}\right)<0
$$


in accordance with essential requirement of the method [60]. Solving (6) and (12), we get the energy equation:

$$
\begin{aligned}
& \left(c_{2}-c_{3}\right) n+c_{3} n^{2}-(2 n+1) c_{5}+c_{7}+2 c_{3} c_{8} \\
& +(2 n+1)\left(\sqrt{c_{9}}+c_{3} \sqrt{c_{8}}\right)+2 \sqrt{c_{8} c_{9}}=0
\end{aligned}
$$

for the potential model under consideration.

In regards of the wave functions. We firstly obtain the solution of the differential equation (8) for the weight function $\rho(z)$ as

$$
\rho(z)=z^{c_{10}}\left(1-c_{3} z\right)^{c_{11}}
$$

and hence from (7), the first part of the wave functions can be expressed in the form of the Jacobi polynomials as

$$
y_{n}(z)=P_{n}^{\left(c_{10}, c_{11}\right)}\left(1-2 c_{3} z\right)
$$

where $\operatorname{Re}\left(c_{10}\right)>-1, \operatorname{Re}\left(c_{11}\right)>-1$ and

$$
\begin{aligned}
& c_{10}=c_{1}+2 c_{4}+2 \sqrt{c_{8}}-1, \\
& c_{11}=1-c_{1}-2 c_{4}+\frac{2}{c_{3}} \sqrt{c_{9}}, c_{3} \neq 0
\end{aligned}
$$

The second part of the wave functions (4) can be found from the solution of the differential equation (10) as

$$
\phi(z)=z^{c_{12}}\left(1-c_{3} z\right)^{c_{13}}
$$

where

$$
c_{12}=c_{4}+\sqrt{c_{8}}, c_{13}=-c_{4}+\frac{1}{c_{3}}\left(\sqrt{c_{9}}-c_{5}\right)
$$

Hence, the general wave functions (4) read as

$$
u_{l}(z)=N_{n l} z^{c_{12}}\left(1-c_{3} z\right)^{c_{13}} P_{n}^{\left(c_{10}, c_{11}\right)}\left(1-2 c_{3} z\right)
$$

where $N_{n l}$ is the normalization constant

\section{Bound-State Solutions}

The $D$-dimensional time-independent arbitrary $l$-state radial $\mathrm{KG}$ equation with scalar and vector potentials $S(r)$ and $V(r)$, respectively, where $\boldsymbol{r}=|\boldsymbol{r}|$ describing a spineless particle takes the general form $[3,62]$ :

$$
\begin{gathered}
\nabla_{D}^{2} \psi_{l_{1}, \cdots, l_{D-2}}^{\left(l_{D-1}=l\right)}(\hat{x})+\frac{1}{\hbar^{2} c^{2}} \\
\times\left\{\left[E_{n l}-V(r)\right]^{2}-\left[M c^{2}+S(r)\right]^{2}\right\} \nabla_{D}^{2} \psi_{l_{1}, \cdots, l_{D-2}}^{\left(l_{D-1}=l\right)}=0 \\
\nabla_{D}^{2}=\sum_{j=1}^{D} \frac{\partial^{2}}{\partial x_{j}^{2}} \\
\psi_{l_{1}, \cdots, l_{D-2}}^{\left(l_{D-1}=l\right)}(\hat{x})=R_{l}(r) Y_{l_{1}, \cdots, l_{D-2}}^{\left(l_{D-1}=l\right)}\left(\theta_{1}, \theta_{2}, \cdots, \theta_{D-1}\right)
\end{gathered}
$$

where $E_{n l}, M$ and $\nabla_{D}^{2}$ stand for KG energy, mass and $D$-dimensional Laplacian, respectively. In addition, $x$ is a
$D$-dimensional position vector. Let us decompose the radial wave function $R_{l}(r)$ as follows:

$$
R_{l}(r)=r^{-(D-1) / 2} u_{l}(r)
$$

we, then, reduce (30a) into the $D$-dimensional radial Schrödinger-like equation with arbitrary orbital angular momentum number $l$ as

$$
\begin{aligned}
& \frac{\mathrm{d}^{2} u_{l}(r)}{\mathrm{d} r^{2}}+\frac{1}{\hbar^{2} c^{2}}\left\{\left[E_{n l}-V(r)\right]^{2}-\left[M_{c}+S(r)\right]^{2}\right. \\
& \left.-\frac{l^{\prime}\left(l^{\prime}+1\right) \hbar^{2} c^{2}}{r^{2}}\right\} u_{l}(r)=0
\end{aligned}
$$

where we have set $l^{\prime}\left(l^{\prime}+1\right)=\left[(\tilde{M}-2)^{2}-1\right] / 4$ and $\tilde{M}=D+2 l$ where $l=0,1,2, \cdots$. Under the equally mixed potentials $S(r)= \pm V(r)$, the $\mathrm{KG}$ turns into a Schrödinger-like equation and thus the bound state solutions are very easily obtained with the help of the wellknown methods developed in the non-relativistic quantum mechanics. We use the existing approximation for the centrifugal potential term in the non-relativistic model $[9,19]$ which is valid only for $q=1$ value [62, 68]:

$$
\begin{aligned}
& \tilde{V}(r)=\frac{l^{\prime}\left(l^{\prime}+1\right)}{r^{2}} \approx 4 \alpha^{2} l^{\prime}\left(l^{\prime}+1\right) \frac{\mathrm{e}^{-2 \alpha r}}{\left(1-q \mathrm{e}^{-2 \alpha r}\right)}, \\
& l^{\prime}=(\tilde{M}-3) / 2,
\end{aligned}
$$

in the limit of small $\alpha$ and $l^{\prime}$.

\subsection{The Eckart-Type Model}

At first, let us rewrite Equation (2) in a form to include the Hulthén potential,

$$
V(r, q)=4 V_{1} \frac{\mathrm{e}^{-2 \alpha r}}{\left(1-q \mathrm{e}^{-2 \alpha r}\right)^{2}}-V_{2} \frac{1}{1-q \mathrm{e}^{-2 \alpha r}}-V_{3} \frac{q \mathrm{e}^{-2 \alpha r}}{1-q \mathrm{e}^{-2 \alpha r}}
$$

and then follow the model used in $[62,68,69]$ by inserting the above equation and the approximate potential term (33) into (32), we obtain

$$
\left.\begin{array}{l}
\frac{\mathrm{d}^{2} u_{l}(r)}{\mathrm{d} r^{2}}+\frac{1}{\hbar^{2} c^{2}} \\
\times\left\{-\frac{\left[8\left(E_{n l} \pm M c^{2}\right) V_{1}+4 \alpha^{2} \hbar^{2} c^{2} l^{\prime}\left(l^{\prime}+1\right)\right] \mathrm{e}^{-2 \alpha r}}{\left(1-q \mathrm{e}^{-2 \alpha r}\right)^{2}}\right. \\
\left.+\frac{2\left(E_{n l} \pm M c^{2}\right)\left(V_{2}+q V_{3} \mathrm{e}^{-2 \alpha r}\right)}{\left(1-q \mathrm{e}^{-2 \alpha r}\right)}\right\} u_{l}(r) \\
=\frac{1}{\hbar^{2} c^{2}}\left[\left(M c^{2}\right)^{2}-E_{n l}^{2}\right] u_{l}(r), u_{l}(0)=0
\end{array}\right\}
$$


which is now amenable to the NU solution. We further use the following ansätze in order to make the above differential equation more compact

$$
\left.\begin{array}{ll}
z(r)=e^{-2 \alpha r}, & Q=2 \hbar c \alpha, \\
\varepsilon_{\mathrm{nl}}=\frac{\sqrt{\left(M c^{2}\right)^{2}-E_{n l}^{2}}}{Q}, & g=\frac{\left(E_{n l} \pm M c^{2}\right)}{Q^{2}} \\
\beta=8 g V_{1}+l^{\prime}\left(l^{\prime}+1\right), & \lambda=2 g V_{3}
\end{array}\right\}
$$

Notice that $\left|E_{n l}\right| \leq M c^{2}$. The KG equation can then be reduced to

$$
\begin{aligned}
& \left\{\frac{\mathrm{d}^{2}}{\mathrm{~d} z^{2}}+\frac{(1-q z)}{z(1-q z)} \frac{d}{d z}+\frac{\left[-A z^{2}+B z-C\right]}{z^{2}(1-q z)^{2}}\right\} u_{l}(z)=0, \\
& A=q^{2}\left(\varepsilon_{n l}^{2}+\lambda\right), B=q\left(2 \varepsilon_{n l}^{2}+\lambda-\gamma-\frac{\beta}{q}\right) \\
& C=\varepsilon_{n l}^{2}-\gamma
\end{aligned}
$$

where $r \in[0, \infty) \rightarrow z \in[0,1]$. Before proceeding, the boundary conditions on the radial wave functions are: $u_{l}(r \rightarrow \infty$, or $z \rightarrow 0) \rightarrow 0$ and $u_{l}(r=0$ or $z=1)$ is finite. Comparing (37) with (13), we obtain values for the set of parameters given in Section 2:

$$
\begin{aligned}
& c_{1}=1, \quad c_{2}=c_{3}=q, \quad c_{4}=0, \quad c_{5}=-\frac{q}{2} \\
& c_{6}=q^{2}\left(\varepsilon_{n l}^{2}+\lambda+\frac{1}{4}\right), c_{7}=-q\left(2 \varepsilon_{n l}^{2}+\lambda-\gamma-\frac{\beta}{q}\right), \\
& c_{8}=\varepsilon_{n l}^{2}-\gamma, c_{9}=\left(\frac{q}{2}\right)^{2}\left(1+\frac{4 \beta}{q}\right), c_{10}=2 \sqrt{\varepsilon_{n l}^{2}-\gamma}, \\
& c_{11}=\sqrt{1+\frac{4 \beta}{q}}, c_{12}=\sqrt{\varepsilon_{n l}^{2}-\gamma}, \\
& c_{13}=\delta=\frac{1}{2}\left(1+\sqrt{1+\frac{4 \beta}{q}}\right)
\end{aligned}
$$

and also the energy equation through (23) as

$$
\varepsilon_{n l}^{2}=\frac{(\gamma+\lambda)^{2}}{4(n+\delta)^{2}}+\frac{(n+\delta)^{2}}{4}+\frac{\gamma-\lambda}{2}, n=0,1,2, \cdots
$$

Making use of (36), the above equation can be rewritten as

$$
\begin{aligned}
M^{2} c^{4}-E_{n l}^{2}= & (\hbar c \alpha)^{2}(n+w)^{2}+\frac{\left(E_{n l} \pm M c^{2}\right)}{(2 \hbar c \alpha)^{2}} \frac{\left(V_{2}+V_{3}\right)^{2}}{(n+w)^{2}} \\
& +\left(E_{n l} \pm M c^{2}\right)\left(V_{2}-V_{3}\right), \\
w= & \frac{1}{2}\left(1+\sqrt{1+\frac{4 l^{\prime}\left(l^{\prime}+1\right)}{q}+\frac{8\left(E_{n l} \pm M c^{2}\right) V_{1}}{q(\hbar c \alpha)^{2}}}\right)
\end{aligned}
$$

The energy $E_{n l}$ is defined implicitly by (40) which is a rather complicated transcendental equation having many solutions for given values of $n$ and $l$ In the above equation, let us remark that it is not difficult to conclude that bound-states appear in four energy solutions; only two energy solutions are valid for the particle $E^{p}=E_{n l}^{+}$ and the second one corresponds to the anti-particle energy $E^{a}=E_{n l}^{-}$in the Eckart-type field.

Referring to the general parametric model in Section 2, we turn to the calculation of the corresponding wave functions. The explicit form of the weight function becomes

$$
\begin{aligned}
& \rho(z)=z^{2 p}(1-q z)^{2 w-1}, \\
& p=\frac{1}{2}\left[n+w-\frac{\left(E_{n l} \pm M c^{2}\right)\left(V_{2}+V_{3}\right)}{2(\hbar c \alpha)^{2}} \frac{1}{(n+w)}\right]
\end{aligned}
$$

which gives the first part of the wave functions in the form of the Jacobi polynomials:

$$
y_{n}(z) \rightarrow P_{n}^{(2 p, 2 w-1)}(1-2 q z)
$$

Further, the second part of the wave functions can be found as

$$
\phi(z) \rightarrow z^{p}(1-q z)^{w}
$$

Hence, the un-normalized wave functions expressed in terms of the Jacobi polynomials read

$$
u_{l}(z)=N_{n l} z^{p}(1-q z)^{w} P_{n}^{(2 p, 2 w-1)}(1-2 q z)
$$

and consequently the total radial part of the wave functions expressed in terms of the hypergeometric functions are

$$
\begin{aligned}
R_{l}(r)= & N_{n l} r^{-(D-1) / 2}\left(e^{-2 \alpha r}\right)^{p}\left(1-q e^{-2 \alpha r}\right)^{w} \\
& \times{ }_{2} F_{1}\left(-n, n+2(p+w) ; 2 p+1 ; q e^{-2 \alpha r}\right)
\end{aligned}
$$

where $N_{n l}$ is a constant related to the normalization. The relationship between the Jacobi polynomials and the hypergeometric functions is given by

$$
P_{n}^{(a, b)}(1-2 q x)={ }_{2} F_{1}(-n, n+a+b+1 ; a+1 ; x)
$$

where

$$
{ }_{2} F_{1}(v, \mu ; \gamma ; x)=\frac{\Gamma(\gamma)}{\Gamma(v) \Gamma(\mu)} \sum_{k=0}^{\infty} \frac{\Gamma(v+k) \Gamma(\mu+k)}{\Gamma(\gamma+k)} \frac{x^{k}}{k !}
$$

Now, in taking $V_{2}=V_{3}$, the energy Equation (40) satisfying $E_{n l}$ for the equally-mixed scalar and vector Eckart-type potentials becomes

$$
M^{2} c^{4}-E_{n l}^{2}=(\hbar c \alpha)^{2}(n+w)^{2}+\frac{\left(E_{n l} \pm M c^{2}\right)^{2}}{(\hbar c \alpha)^{2}} \frac{V_{2}^{2}}{(n+w)^{2}}
$$


and the wave functions:

$$
\begin{aligned}
& u_{l}(z)=N_{n l} z^{v}(1-q z)^{w} P_{n}^{(2 v, 2 w-1)}(1-2 q z), \\
& v=\frac{1}{2}\left[n+w-\frac{\left(E_{n l} \pm M c^{2}\right) V_{2}}{(\hbar c \alpha)^{2}} \frac{1}{(n+w)}\right]
\end{aligned}
$$

or the total radial wave functions in (45) are

$$
\begin{aligned}
R_{l}(r)= & N_{n l} r^{-(D-1) / 2}\left(\mathrm{e}^{-2 \alpha r}\right)^{v}\left(1-q \mathrm{e}^{-2 \alpha r}\right)^{w} \\
& \times{ }_{2} F_{1}\left(-n, n+2(v+w) ; 2 v+1 ; q e^{-2 \alpha r}\right)
\end{aligned}
$$

where $N_{n l}$ is a normalization factor. The results given in (46) and (47) are consistent with those given in (15) and (18) of [58].

Taking $q=1, \quad 2 \alpha \rightarrow \alpha, \quad V_{1}=V_{2}=0 \quad$ and $V_{3}=0$, (34) has become the Hulthén potential. Hence, we find bound state solutions for equally-mixed scalar and vector $S(r)=V(r)$ Hulthén potentials in the $\mathrm{KG}$ theory with any orbital angular quantum number $l$ and an arbitrary dimension $D$,

$$
\begin{aligned}
& \sqrt{M^{2} c^{4}-E_{n l}^{2}}=\frac{(\hbar c \alpha)(n+v)}{2}-\frac{\left(E_{n l}+M c^{2}\right) V_{0}}{(\hbar c \alpha)} \frac{1}{(n+v)}, \\
& v=\frac{D+2 l-1}{2}
\end{aligned}
$$

and

$$
\begin{aligned}
& u_{l}(z)=N_{n l}\left(\mathrm{e}^{-\alpha r}\right)^{\varsigma}\left(1-\mathrm{e}^{-\alpha r}\right)^{v} P_{n}^{(2 \varsigma, 2 v-1)}(1-2 z), \\
& \varsigma=\frac{n+v}{2}-\frac{\left(E_{n l}+M c^{2}\right) V_{0}}{(\hbar c \alpha)^{2}} \frac{1}{n+v}
\end{aligned}
$$

The Jacobi polynomial in the above equation can be expressed in terms of the hypergeometric function:

$$
\begin{aligned}
R_{l}(r)= & N_{n l} r^{-(D-1) / 2}\left(\mathrm{e}^{-\alpha r}\right)^{\varsigma}\left(1-\mathrm{e}^{-\alpha r}\right)^{v} \\
& \times{ }_{2} F_{1}\left(-n, n+2(\varsigma+v) ; 2 \varsigma+1 ; \mathrm{e}^{-\alpha r}\right)
\end{aligned}
$$

where $N_{n l}$ is a constant related to the normalization. The above results are identical to those found recently by [62,70].

In the non-relativistic limit, inserting the equally mixed Eckart-type potentials (1) into the Schrödinger equation gives

$$
\begin{aligned}
& \frac{\mathrm{d}^{2} u_{l}(r)}{\mathrm{d} r^{2}}+\left\{\frac{2 M E_{n l}}{\hbar^{2}}-\frac{\left[8 M V_{1}+4 \alpha^{2} \hbar^{2} l^{\prime}\left(l^{\prime}+1\right)\right]}{\hbar^{2}\left(1-q \mathrm{e}^{-2 \alpha r}\right)^{2}} \mathrm{e}^{-2 \alpha r}\right. \\
& \left.+\frac{2 M V_{2}\left(1+q \mathrm{e}^{-2 \alpha r}\right)}{\hbar^{2}\left(1-q \mathrm{e}^{-2 \alpha r}\right)}\right\} u_{l}(r)=0
\end{aligned}
$$

and further making use of the following definitions:

$$
\begin{aligned}
& \varepsilon_{n l}=\frac{\sqrt{-2 M E_{n l}}}{T}, E_{n l} \leq 0 \quad \beta=\frac{8 M V_{1}}{T^{2}}+l^{\prime}\left(l^{\prime}+1\right), \\
& \gamma=\frac{2 M V_{2}}{T^{2}}, T=2 \hbar \alpha
\end{aligned}
$$

lead us to obtain the set of parameters and energy equation given before in (38) and (39) with $\gamma=\lambda$. Incorporating the above equation and using (39), we find the following energy eigenvalues:

$$
\begin{aligned}
& E_{n l}=-\frac{1}{2 M}\left[\hbar^{2} \alpha^{2}\left(n+w_{1}\right)^{2}+\frac{M^{2} V_{2}^{2}}{\hbar^{2} \alpha^{2}} \frac{1}{\left(n+w_{1}\right)^{2}}\right] \\
& w_{1}=\frac{1}{2}\left(1+\sqrt{\left(1+2 l^{\prime}\right)^{2}+\frac{8 M V_{1}}{\hbar^{2} \alpha^{2}}}\right)
\end{aligned}
$$

In addition, following procedures indicated in (41) (45), we obtain expressions for the radial wave functions:

$$
\begin{aligned}
R_{l}(r)= & N_{n l} r^{-(D-1) / 2}\left(\mathrm{e}^{-2 \alpha r}\right)^{p_{1}}\left(1-\mathrm{e}^{-2 \alpha r}\right)^{w_{1}} \\
& \times P_{n}^{\left(2 p_{1}, 2 w_{1}-1\right)}\left(1-2 \mathrm{e}^{-2 \alpha r}\right), \\
p_{1}= & \frac{1}{2 \hbar \alpha} \sqrt{-2 M\left(E_{n l}+V_{2}\right)} \\
=\frac{1}{2} & {\left[n+w_{1}-\frac{M V_{2}}{(\hbar \alpha)^{2}} \frac{1}{n+w_{1}}\right] }
\end{aligned}
$$

\subsection{The Rosen-Morse-Type Model}

Under the replacement of $q$ by $-q$, the Eckart-type potential model (1) will become the Rosen-Morse-type potential model given in (2) of Ref. [52]:

$$
\begin{aligned}
& V(r, q)=V_{1} \sec h_{q}^{2}(\alpha r)-V_{2} \tanh _{q}(\alpha r), \\
& V_{1}, V_{2}>0
\end{aligned}
$$

or alternatively [39,40,72]

$$
V(r, q)=4 V_{1} \frac{\mathrm{e}^{-2 \alpha r}}{\left(1+q \mathrm{e}^{-2 \alpha r}\right)^{2}}-V_{2} \frac{1-q \mathrm{e}^{-2 \alpha r}}{1+q \mathrm{e}^{-2 \alpha r}}
$$

We may rewrite the above equation in a form to include the Woods-Saxon potential,

$$
\begin{aligned}
V(r, q)= & 4 V_{1} \frac{\mathrm{e}^{-2 \alpha r}}{\left(1+q \mathrm{e}^{-2 \alpha r}\right)^{2}}-V_{2} \frac{1}{1+q \mathrm{e}^{-2 \alpha r}} \\
& +V_{3} \frac{q \mathrm{e}^{-2 \alpha r}}{1+q \mathrm{e}^{-2 \alpha r}}
\end{aligned}
$$

Defining the parameters: 


$$
\left.\begin{array}{l}
\varepsilon_{\mathrm{n} 0}=\frac{\sqrt{\left(M c^{2}\right)^{2}-E_{n 0}^{2}}}{Q}, g=\frac{\left(E_{n 0} \pm M c^{2}\right)}{Q^{2}} \\
\tilde{\beta}=\beta(l \rightarrow 0)=8 g V_{1}, \\
\tilde{\gamma}=\gamma(l \rightarrow 0)=2 g V_{2}, \\
\tilde{\lambda}=\lambda(l \rightarrow 0)=2 g V_{3}
\end{array}\right\}
$$

we can easily write the s-wave KG equation with $S(r)=$ $\pm V(r)$ for the potential (58) as

$$
\begin{aligned}
& \left\{\frac{\mathrm{d}^{2}}{\mathrm{~d} z^{2}}+\frac{(1+q z)}{z(1+q z)} \frac{\mathrm{d}}{\mathrm{d} z}+\frac{\left[-A z^{2}+B z-C\right]}{z^{2}(1-q z)^{2}}\right\} u_{n}(z)=0, \\
& A=q^{2}\left(\varepsilon_{n 0}^{2}+\tilde{\lambda}\right), B=q\left(\tilde{\gamma}-\tilde{\lambda}-2 \varepsilon_{n l}^{2}-\frac{\tilde{\beta}}{q}\right), \\
& C=\varepsilon_{n 0}^{2}-\tilde{\gamma}
\end{aligned}
$$

Following the steps of solution mentioned in the previous subsection, we may obtain values for the parameters given in Section 2:

$$
\begin{aligned}
& c_{1}=1, \quad c_{2}=c_{3}=-q, \quad c_{4}=0, \quad c_{5}=\frac{q}{2} \\
& c_{6}=q^{2}\left(\varepsilon_{n 0}^{2}+\tilde{\lambda}+\frac{1}{4}\right), c_{7}=q\left(2 \varepsilon_{n 0}^{2}+\tilde{\lambda}-\tilde{\gamma}+\frac{\tilde{\beta}}{q}\right), \\
& c_{8}=\varepsilon_{n 0}^{2}-\tilde{\gamma}, c_{9}=\left(\frac{q}{2}\right)^{2}\left(1-\frac{4 \tilde{\beta}}{q}\right), \quad c_{10}=2 \sqrt{\varepsilon_{n 0}^{2}-\tilde{\gamma}} \\
& c_{11}=\sqrt{1-\frac{4 \tilde{\beta}}{q}}, c_{12}=\sqrt{\varepsilon_{n 0}^{2}-\tilde{\gamma}} \\
& c_{13}=\tilde{\delta}=\frac{1}{2}\left(1-\sqrt{1-\frac{4 \tilde{\beta}}{q}}\right)
\end{aligned}
$$

and the energy equation

$$
\varepsilon_{n 0}^{2}=\frac{(\tilde{\gamma}+\tilde{\lambda})^{2}}{4(n+\tilde{\delta})^{2}}+\frac{(n+\tilde{\delta})^{2}}{4}+\frac{\tilde{\gamma}-\tilde{\lambda}}{2}, n=0,1,2, \cdots
$$

Inserting (59) in the above equation, we obtain energy equation satisfying $E_{n 0}$,

$$
\begin{aligned}
& M^{2} c^{4}-E_{n 0}^{2}=(\hbar c \alpha)^{2}(n+\tilde{w})^{2}+\frac{\left(E_{n 0} \pm M c^{2}\right)^{2}}{(2 \hbar c \alpha)^{2}} \frac{\left(V_{2}+V_{3}\right)^{2}}{(n+\tilde{w})^{2}} \\
&+\left(E_{n 0} \pm M c^{2}\right)\left(V_{2}-V_{3}\right), \\
& \tilde{w}=\frac{1}{2}\left(1-\sqrt{1-\frac{8\left(E_{n 0} \pm M c^{2}\right) V_{1}}{q(\hbar c \alpha)^{2}}}\right)
\end{aligned}
$$

The corresponding un-normalized wave functions can be calculated as before. The explicit form of the weight function reads

$$
\begin{aligned}
& \rho(z)=z^{2 \tilde{p}}(1-q z)^{2 \tilde{w}-1}, \\
& \tilde{p}=\frac{1}{2}\left[n+\tilde{w}-\frac{\left(E_{n 0} \pm M c^{2}\right)\left(V_{2}+V_{3}\right)}{2(\hbar c \alpha)^{2}} \frac{1}{(n+\tilde{w})}\right]
\end{aligned}
$$

which gives the Jacobi polynomials

$$
y_{n}(z) \rightarrow P_{n}^{(2 \tilde{p}, 2 \tilde{w}-1)}(1+2 q z)
$$

as the first part of the wave function. The second part of the wave function can be found as

$$
\phi(z) \rightarrow z^{\tilde{p}}(1+q z)^{\tilde{w}}
$$

Hence, the un-normalized wave function reads

$$
u_{n}(z)=\tilde{N}_{n} z^{\tilde{p}}(1+q z)^{\tilde{w}} P_{n}^{(2 \tilde{p}, 2 \tilde{w}-1)}(1+2 q z)
$$

and thus the total radial part of the radial wave functions in (30) can be expressed in terms of the hypergeometric functions as

$$
\begin{aligned}
R_{n}(r)= & \tilde{N}_{n}\left(\mathrm{e}^{-2 \alpha r}\right)^{\tilde{p}}\left(1+q \mathrm{e}^{-2 \alpha r}\right)^{\tilde{w}} \\
& \times{ }_{2} F_{1}\left(-n, n+2(\tilde{p}+\tilde{w}) ; 2 \tilde{p}+1 ;-q \mathrm{e}^{-2 \alpha r}\right)
\end{aligned}
$$

where $\tilde{N}_{n}$ is a normalization factor.

Taking $V_{2}=V_{3}$ in (63), we find the equation for the potential in (56) satisfying $E_{n 0}$ in the s-wave KG theory,

$$
\begin{aligned}
& M^{2} c^{4}-E_{n 0}^{2} \\
= & (\hbar c \alpha)^{2}(n+\tilde{w})^{2}+\frac{\left(E_{n 0} \pm M c^{2}\right)^{2}}{(\hbar c \alpha)^{2}} \frac{V_{2}^{2}}{(n+\tilde{w})^{2}}
\end{aligned}
$$

and the wave functions take the form

$$
\begin{aligned}
u_{n}(r)= & \tilde{N}_{n}\left(\mathrm{e}^{-2 \alpha r}\right)^{\tilde{p}_{1}}\left(1+q \mathrm{e}^{-2 \alpha r}\right)^{\tilde{w}} \\
& \times{ }_{2} F_{1}\left(-n, n+2\left(\tilde{p}_{1}+\tilde{w}\right) ; 2 \tilde{p}_{1}+1 ;-q e^{-2 \alpha r}\right) \\
\tilde{p}_{1}=\frac{1}{2} & {\left[n+\tilde{w}-\frac{\left(E_{n 0} \pm M c^{2}\right) V_{2}}{(\hbar c \alpha)^{2}} \frac{1}{(n+\tilde{w})}\right], }
\end{aligned}
$$

where $\tilde{N}_{n}$ is a normalization constant. After the following mapping on the potential parameter: $V_{1} \rightarrow-V_{1}$ in (56), the results in (69) and (70) become identical with (13) and (14) of [52].

Also, taking $q=1,2 \alpha \rightarrow \alpha, V_{1}=V_{2}=0$ and $V_{3}=-V_{0}$, (58) turns to become the Woods-Saxon potential. Hence, we can find bound state solutions in the $s$-wave KG theory with equally-mixed scalar and vector $S(r)=V(r)$ for Woods-Saxon potentials as 


$$
\sqrt{M^{2} c^{4}-E_{n 0}^{2}}=\hbar c \alpha \tilde{p}_{2}, \quad \tilde{p}_{2}=\frac{n}{2}+\frac{\left(E_{n 0}+M c^{2}\right)}{(\hbar c \alpha)^{2}} \frac{V_{0}}{n},
$$

and wave functions:

$$
u_{n}(r)=N_{n}\left(\mathrm{e}^{-\alpha r}\right)^{\tilde{p}_{2}} P_{n}^{\left(2 \tilde{p}_{2},-1\right)}\left(1+2 \mathrm{e}^{-\alpha r}\right),
$$

or alternatively, it can be expressed in terms of the hypergeometric function as

$$
R_{n}(r)=N_{n}\left(\mathrm{e}^{-\alpha r}\right)^{\tilde{p}_{2}}{ }_{2} F_{1}\left(-n, n+2 \tilde{p}_{2} ; 2 \tilde{p}_{2}+1 ; \mathrm{e}^{-\alpha r}\right)
$$

where $N_{n}$ is a constant related to the normalization. Under appropriate parameter replacements, we obtain the non-relativistic limit of the energy eigenvalues and eigenfunctions of the above two equations are

$$
E_{n 0}=-\frac{1}{2 M}\left[\frac{n \hbar \alpha}{2}+\frac{2 M V_{0}}{\hbar \alpha} \frac{1}{n}\right], n \neq 0,
$$

and

$$
\begin{aligned}
& u_{n}(r)=N_{n}\left(\mathrm{e}^{-\alpha r}\right)_{2}^{\tilde{p}_{3}} F_{1}\left(-n, n+2 \tilde{p}_{2} ; 2 \tilde{p}_{2}+1 ; \mathrm{e}^{-\alpha r}\right), \\
& \tilde{p}_{3}=\frac{n}{2}+\frac{2 M V_{0}}{(\hbar c \alpha)^{2}} \frac{1}{n},
\end{aligned}
$$

respectively, which is simply the solution of the Schrödinger equation for the potential $\Sigma(r)=V(r)$ $+S(r)=2 V(r)$. The above results are identical to those found before in [8].

\section{Discussions}

In this section, at first, we choose appropriate parameters in the Eckart-type potential model to construct the Eckart potential, Rosen-Morse well and their PT-symmetric versions, and then discuss their energy equations in the framework of KG theory with equally mixed potentials.

\subsection{Eckart Potential Model}

Taking $q=1$, the potential (1) turns to the standard Eckart potential [41]

$$
V(r)=V_{1} \operatorname{cosech}(\alpha r)-V_{2} \operatorname{coth}(\alpha r), V_{1}, V_{2}>0
$$

In natural units $(\hbar=c=1)$, we can obtain the energy equation (46) for the Eckart potential in $3 D$ space spinless KG theory as

$$
\begin{aligned}
& M^{2}-E_{n l}^{2}=\alpha^{2}\left(n+w^{\prime}\right)^{2}+\frac{V_{2}^{2}\left(E_{n l} \pm M\right)^{2}}{\alpha^{2}\left(n+w^{\prime}\right)^{2}}, \\
& w^{\prime}=w(q \rightarrow 1)=\frac{1}{2}\left(1+\sqrt{\left(2 l^{\prime}+1\right)^{2}+\frac{8\left(E_{n l} \pm M\right) V_{1}}{\alpha^{2}}}\right),
\end{aligned}
$$

which is identical with those given in Equation (22) of [52] under the equally-mixed potential restriction given by $S(r)= \pm V(r)$. The unnormalized wave function corresponding to the energy levels is

$$
\begin{aligned}
R_{l}(r)= & N_{n l} r^{-(D-1) / 2}\left(\mathrm{e}^{-2 \alpha r}\right)^{v}\left(1-\mathrm{e}^{-2 \alpha r}\right)^{w^{\prime}} \\
& \times{ }_{2} F_{1}\left(-n, n+2\left(v+w^{\prime}\right) ; 2 v+1 ; \mathrm{e}^{-2 \alpha r}\right),
\end{aligned}
$$

where $N_{n l}$ is a normalization factor.

1) For $s$-wave case, the centrifugal term

$$
(D+2 l-1)(D+2 l-3) /\left(4 r^{2}\right)=0
$$

and hence

$$
(D+2 l-1)(D+2 l-3) \alpha^{2} \mathrm{e}^{-2 \alpha r} /\left(1-e^{-2 \alpha r}\right)^{2}=0
$$

too. Thus, the energy eigenvalues take the following simple form

$$
\begin{aligned}
& M^{2}-E_{n 0}^{2}=\alpha^{2}\left(n+w_{1}\right)^{2}+\frac{V_{2}^{2}\left(E_{n 0} \pm M\right)^{2}}{\alpha^{2}\left(n+w_{1}\right)^{2}}, \\
& w_{1}=\frac{1}{2}\left(1+\sqrt{1+\frac{8\left(E_{n 0} \pm M\right) V_{1}}{\alpha^{2}}}\right) .
\end{aligned}
$$

2) In the non-relativistic approximation of the $K G$ energy equation (potential energies small compared to $M c^{2}$ and $E \approx M c$ Equation (32) reduces into the form [72]

$$
\begin{aligned}
& -\frac{\hbar^{2}}{2 M} \frac{\mathrm{d}^{2} u_{l}(r)}{\mathrm{d} r^{2}}+\left\{V(r)+S(r)-\frac{l^{\prime}\left(l^{\prime}+1\right) \hbar^{2}}{r^{2}}\right\} \times u_{l}(r) \\
= & \left(E_{n l}-M c^{2}\right) u_{l}(r) .
\end{aligned}
$$

When $V(r)=S(r)$, the energy spectrum obtained from (80) reduces to those energy spectrum obtained from the solution of the Schrödinger equation for the sum potential $\Sigma(r)=2 V(r)$. In other words, the nonrelativistic limit is the Schrödinger-like equation for the potential

$$
8 V_{1} \frac{\mathrm{e}^{-2 \alpha r}}{\left(1-\mathrm{e}^{-2 \alpha r}\right)^{2}}-2 V_{2} \frac{\left(1+\mathrm{e}^{-2 \alpha r}\right)}{\left(1-\mathrm{e}^{-2 \alpha r}\right)} .
$$

This can be achieved by making the parameter replacements $E_{R}+M \rightarrow 2 M$ and $E_{R}-M \rightarrow E_{N R}$, so the non-relativistic limit of our results in (46) reduces to

$$
E_{N R}=-\frac{1}{2 M}\left[\alpha^{2}\left(n+w_{2}\right)^{2}+\frac{2 M^{2} V_{2}^{2}}{\alpha^{2}\left(n+w_{2}\right)^{2}}\right],
$$

and the corresponding wave functions in (48) become 


$$
\begin{aligned}
R_{l}(r)= & N_{n l} r^{-(D-1) / 2}\left(\mathrm{e}^{-2 \alpha r}\right)^{v_{2}}\left(1-\mathrm{e}^{-2 \alpha r}\right)^{w_{2}} \\
& \times{ }_{2} F_{1}\left(-n, n+2\left(v_{2}+w_{2}\right) ; 2 v_{2}+1 ; \mathrm{e}^{-2 \alpha r}\right), \\
v_{2}=\frac{1}{2} & {\left[n+w_{2}-\frac{2 M V_{2}}{\alpha^{2}\left(n+w_{2}\right)}\right], } \\
w_{2}=\frac{1}{2} & {\left[1+\sqrt{\left(2 l^{\prime}+1\right)^{2}+\frac{16 M V_{1}}{\alpha^{2}}}\right] . }
\end{aligned}
$$

The above two equations are identical with the NU solution of the Schrödinger equation for a potential $V(r)$ (cf. $[68,69])$.

\subsection{PT-Symmetric Trigonometric Rosen-Morse (tRM) Potential}

When we make the transformations of parameters as $\alpha \rightarrow i \alpha, \quad V_{2}=-i V_{2}$ and $V_{1} \rightarrow-V_{1}$, and further using the relation between the trigonometric and the hyperbolic functions $\sin (i \alpha x)=i \sinh (\alpha x)$, the potential (1) turns to become the PT-symmetric tRM potential [73]:

$$
V(x)=V_{1} \csc ^{2}(\alpha x)-V_{2} \cot (\alpha x), \operatorname{Re}\left(V_{1}\right)>0
$$

where $\quad \alpha=\pi /(2 d), \quad x=(0, d], \quad V_{1}=a(a+1) \quad$ and $V_{2}=2 b$. This potential is displayed in Figure 1 which is nearly linear in $\pi / 3<\alpha x<2 \pi / 3$, Coulombic in $\pi / 90$ $<\alpha x<\pi / 30$ and infinite walls at 0 and $\pi$ So it might be a prime candidate for an effective QCD potential. For a potential $V(x)$, when one makes the transformation of $x \rightarrow-x$ and $i \rightarrow-i$, if the relation $V(-x)=V^{*}(x)$ exists, the potential $V(x)$ is said to be PT-symmetric, where $P$ denotes parity operator (space reflection) and $T$ denotes time reversal $[8,74]$. Our point here is that $V(x)$ interpolates between the Coulomb-and the infinite wall potential [75] going through an intermediary

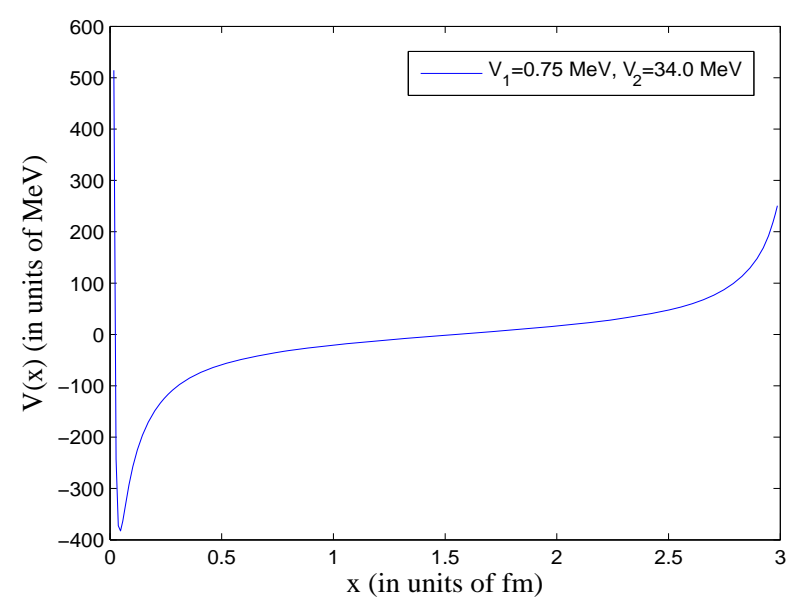

Figure 1. Plot of the tRM potential [see (83)] for a set of parameters $a=0.5$ and $b=\mathbf{1 7 . 0}$. region of linear- $x$ and harmonic-oscillator $x^{2}$ dependences. To see this it is quite instructive to expand the potential in a Taylor series which for appropriately small $x$ takes the form of a Coulomb-like potential with a centrifugal-barrier like term, provided by the $\csc ^{2}(\alpha x)$ part [76],

$$
V(x) \approx \frac{V_{2}}{\alpha x}+\frac{V_{1}}{(\alpha x)^{2}}, \alpha x \ll 1,
$$

For $\alpha x<\pi / 2$ we can then take the potential (84) plus a linear like perturbation

$$
\Delta V(x)=\frac{V_{1}}{3}+\frac{V_{2}}{3} x
$$

as an approximation of tRM potential. The potential (83) obviously evolves to an infinite wall as $\alpha x$ approaches the limits of the definition interval $0<\alpha x<\pi$, due to the behavior of the $\cot \alpha x$ and $\csc \alpha x$ for $V_{1}>0$. The potential is essential for the QCD quark-gluon dynamics where the one gluon exchange gives rise to an effective Coulomb-like potential, while the self gluon interactions produce a linear potential as established by lattice QCD calculations of hadron properties (Cornell potential) [77]. Finally, the infinite wall piece of the tRM potential provides the regime suited for the asymptotical freedom of the quarks. Now, making the corresponding parameter replacements in (46), we end up with real energy equation for the above PT-symmetric version of the Eckart-type potentialş in the $\mathrm{KG}$ equation with equally mixed potentials,

$$
\left(M c^{2}\right)^{2}-E_{n l}^{2}=\frac{\left(E_{n l} \pm M c^{2}\right)^{2} V_{2}^{2}}{(\hbar c \alpha)^{2}(n+w)^{2}}-(\hbar c \alpha)^{2}(n+w)^{2},
$$

and the radial wave functions build up as

$$
\begin{aligned}
& R_{l}(x)= N_{n l} x^{-(D-1) / 2}\left(\mathrm{e}^{i 2 \alpha r}\right)^{v}\left(1-\mathrm{e}^{i 2 \alpha r}\right)^{w} \\
& \times{ }_{2} F_{1}\left(-n, n+2(v+w) ; 2 v+1 ; \mathrm{e}^{i 2 \alpha r}\right), \\
& v=\frac{1}{2}\left[n+w+i \frac{\left(E_{n l} \pm M c^{2}\right) V_{2}}{(\hbar c \alpha)^{2}(n+w)}\right], \\
& w=\frac{1}{2}\left[1+\sqrt{\left(2 l^{\prime}+1\right)^{2}+\frac{8\left(E_{n l} \pm M c^{2}\right) V_{1}}{(\hbar c \alpha)^{2}}}\right] .
\end{aligned}
$$

\subsection{Standard Rosen-Morse Well}

Taking $q=1, V_{1} \rightarrow-V_{1}(\tilde{\beta} \rightarrow-\tilde{\beta})$, and $V_{2} \rightarrow-V_{2}(\tilde{\gamma} \rightarrow-\tilde{\gamma})$, the potential (56) turns to the standard Rosen-Morse well $[39,71]$ 


$$
V(r)=-V \sec h^{2} \alpha r+V_{2} \tanh \alpha r, V_{1}, V_{2}>0 .
$$

This potential is useful in discussing polyatomic molecular vibrational energies. An example of its application to the vibrational states of $\mathrm{NH}_{3}$ was given by Rosen and Morse in [39,71]. Making the corresponding parameter replacements in Equation (69), we obtain the energy equation for the Rosen-Morse well in the s-wave $\mathrm{KG}$ theory with equally mixed potentials,

$$
\begin{aligned}
& M^{2}-E_{n 0}^{2}=\alpha^{2}\left(n+\tilde{\delta}_{1}\right)^{2}+\frac{V_{2}^{2}\left(E_{n 0} \pm M\right)^{2}}{\alpha^{2}\left(n+\tilde{\delta}_{1}\right)^{2}}, \\
& \tilde{\delta}_{1}=\frac{1}{2}\left(1-\sqrt{1+\frac{8\left(E_{n 0} \pm M\right) V_{1}}{\alpha^{2}}}\right) .
\end{aligned}
$$

The un-normalized wave function corresponding to the energy levels is

$$
u_{n}(r)=\tilde{N}_{n}^{\prime}\left(\mathrm{e}^{-2 \alpha r}\right)^{\tilde{\eta}_{1}}\left(1+\mathrm{e}^{-2 \alpha r}\right)^{\tilde{\delta}_{1}} P_{n}^{\left(2 \tilde{\eta}_{1}, 2 \tilde{\delta}_{1}-1\right)}\left(1+2 \mathrm{e}^{-2 \alpha r}\right),
$$

$$
\tilde{\eta}_{1}=\frac{1}{2}\left[n+\tilde{\delta}_{1}+\frac{\left(E_{n 0} \pm M\right) V_{2}}{\alpha^{2}\left(n+\tilde{\delta}_{1}\right)}\right],
$$

where $\tilde{N}_{n}^{\prime}$ is a normalization constant. The results given in (89) and (90) are consistent with those given in (19) and (20) of [52], respectively. The $s$-wave energy states of the KG equation for the Rosen-Morse potential are calculated for a set of selected values parameters in Table 1.

When $V_{0}=S_{0}$, the non-relativistic limit is the solution of the Schrödinger equation for the potential

\begin{tabular}{|c|c|c|c|c|c|c|c|c|c|}
\hline$n$ & $\alpha$ & $q$ & $V_{1}$ & $V_{2}$ & $M$ & $E_{1}$ & $E_{2}$ & $E_{3}$ & $E_{4}$ \\
\hline 1 & 1 & 1 & 1 & 1 & 4 & $1.8137^{\mathrm{a}}$ & $-1.9140^{\mathrm{a}}$ & $-3.3923^{\mathrm{a}}$ & $-3.9088^{\mathrm{a}}$ \\
\hline 2 & & & & & & -2.2117 & -3.6791 & & \\
\hline 3 & & & & & & -0.6606 & -3.3105 & & \\
\hline 4 & & & & & & 0.8879 & -2.7697 & & \\
\hline 5 & & & & & & 1.8766 & -1.9765 & & \\
\hline 1 & 1 & 1 & 2 & -2 & 5 & 0.9989 & -3.7763 & -4.7275 & -4.9351 \\
\hline 2 & & & & & & -4.1746 & -4.7795 & & \\
\hline 3 & & & & & & -3.3814 & -4.5376 & & \\
\hline 4 & & & & & & -2.3989 & -4.2008 & & \\
\hline 5 & & & & & & -1.3083 & -3.7529 & & \\
\hline 1 & 0.5 & 1 & 1 & -1 & 4 & 1.9558 & -3.5288 & -3.8460 & -3.9773 \\
\hline 2 & & & & & & 1.9608 & -2.5367 & -3.5326 & -3.9216 \\
\hline 3 & & & & & & 1.2294 & -0.5126 & -3.0732 & -3.8358 \\
\hline 4 & & & & & & -2.4823 & -3.7191 & & \\
\hline 5 & & & & & & -1.7822 & -3.5695 & & \\
\hline 1 & 1 & 0.5 & 1 & -1 & 4 & 1.5783 & -3.2245 & -3.6502 & -3.9258 \\
\hline 2 & & & & & & 1.9995 & -1.5367 & -2.9520 & -3.7496 \\
\hline 3 & & & & & & -1.9529 & -3.4736 & & \\
\hline 4 & & & & & & -0.7335 & -3.0839 & & \\
\hline 5 & & & & & & 0.5489 & -2.5528 & & \\
\hline
\end{tabular}

$$
-8 V_{1} \frac{\mathrm{e}^{-2 \alpha r}}{\left(1+\mathrm{e}^{-2 \alpha r}\right)^{2}}+2 V_{2} \frac{\left(1-\mathrm{e}^{-2 \alpha r}\right)}{\left(1+\mathrm{e}^{-2 \alpha r}\right)} .
$$

In the non-relativistic limits, the energy spectrum is

$$
E_{N R}=-\frac{1}{2 M}\left[\alpha^{2}\left(n+\tilde{\delta}_{2}\right)^{2}+\frac{4 M^{2} V_{2}^{2}}{\alpha^{2}\left(n+\tilde{\delta}_{2}\right)^{2}}\right],
$$

Table 1. The s-wave energy spectrum of the equally mixed scalar and vector Rosen-Morse-type potentials.

${ }^{\mathrm{a}}$ The present results are identical to the ones given in [52]. 


$$
\tilde{\delta}_{2}=\frac{1}{2}\left(1-\sqrt{1+\frac{16 M V_{1}}{\alpha^{2}}}\right),
$$

and the wave functions are

$$
\begin{aligned}
& R_{n}(r)= \tilde{N}_{n}^{\prime}\left(\mathrm{e}^{-2 \alpha r}\right)^{\tilde{\eta}_{2}}\left(1+\mathrm{e}^{-2 \alpha r}\right)^{\tilde{\delta}_{2}} P_{n}^{\left(2 \tilde{\eta}_{2}, 2 \tilde{\delta}_{2}-1\right)} \\
& \times\left(1+2 \mathrm{e}^{-2 \alpha r}\right), \\
& \tilde{\eta}_{2}=\frac{1}{2}\left[n+\tilde{\delta}_{2}+\frac{2 M V_{2}}{\alpha^{2}\left(n+\tilde{\delta}_{2}\right)}\right] .
\end{aligned}
$$

\section{Conclusions and Outlook}

A parametric generalization short-cut derived from the NU have been used to carry out the analytic bound states (real energy spectrum and wave functions) of the $\mathrm{KG}$ equation with any orbital quantum number $l$ for equally mixed scalar and vector Eckart-type potentials. The present solutions include energy equation and un-normalized wave functions which have been expressed in terms of the Jacobi polynomials (or hypergeometric functions). Additionally, in making appropriate changes in the Eckarttype potential parameters, one can easily generate new energy spectrum formulas for various types of the wellknown molecular potentials such as the Rosen-Morse well [39], the Eckart potential, the Hulthén potential [13], the Woods-Saxon potential [7] and the Manning-Rosen potential [31] and others. It is also noted that under the PT-symmetry property, the exponential potentials can be reduced to the trigonometric potentials with real bound state solutions. Also, the KG equation with equally mixed scalar and vector Rosen-Morse-type potentials can be solved exactly for s-wave bound states ( $l=0$ case). The calculated energy equations of these potentials are seen to be complicated transcendental equations in the relativistic model [39]. The non-relativistic limit can be easily reached by making a mapping on the parameters and/or solving the original Schrödinger equation. It is found that the relativistic and non-relativistic results are identical with those ones obtained in literature through the various methods.

\section{Acknowledgements}

The author thanks the kind referees for their invaluable suggestions which greatly helped in improving the manuscript.

\section{References}

[1] L. I. Schiff, "Quantum Mechanics," 3rd Edition,
McGraw-Hill, New York, 1955.

[2] L. D. Landau and E. M. Lifshitz, "Quantum Mechanics, Non-Relativistic Theory," 3rd. Edition, Pergamon, Oxford, 1977.

[3] C. L. Pekeris, "The Rotation-Vibration Coupling in Diatomic Molecules," Physical Review, Vol. 45, 1934, pp. 98-103. doi:10.1103/PhysRev.45.98

[4] O. Bayrak and I. Boztosun, "Arbitrary $\ell$-State Solutions of the Rotating Morse Potential by the Asymptotic Iteration Method," Journal of Physics A: Mathematical and General, Vol. 39, No. 22, 2006, pp. 6955-6964. doi:10.1088/0305-4470/39/22/010

[5] W.C. Qiang and S. H. Dong, “Arbitrary l-State Solutionsof the Rotating Morse potential Through the Exact Quantization Method," Physics Letters A, Vol. 363, No.3, 2007, pp. 169-176. doi:10.1016/j.physleta.2006.10.091

[6] S. M. Ikhdair, "An Approximate $\kappa$ State Solutions of the Dirac Equation for the Generalized Morse Potential under Spin and Pseudospin Symmetry," Journal of Mathematical Physics, Vol. 52, No. 5, 2011, pp. 052303052322. doi:10.1063/1.3583553

[7] S. M. Ikhdair and R. Sever, "Any $\ell$-State Solutions of the Woods-Saxon Potential in Arbitrary Dimension within the New Improved Quantization Rule," International Journal of Modern Physics A, Vol. 25, No. 20, 2010, pp. 3941-3952. doi:10.1142/S0217751X10050160

[8] S. M. Ikhdair and R. Sever, "Exact Solution of the Klein-Gordon Equation for the PT-Symmetric Generalized Woods-Saxon Potential by the Nikiforov-Uvarov Method," Annals of Physics (Leibzig), Vol. 16, No. 3, 2007, pp. 218-232. doi:10.1002/andp.200610232

[9] S. M. Ikhdair and R. Sever, "Approximate Eigenvalue and Eigen Function Solutions for the Generalized Hulthén Potential with any Angular Momentum," Journal of Mathematical Chemistry, Vol. 42, No. 3, 2007, pp. 461- 471. doi:10.1007/s10910-006-9115-8

[10] C. Y. Chen, D. S. Sun and F. L. Lu, “Approximate Analytical Solutions of Klein-Gordon Equation with Hulthén Potentials for Nonzero Angular Momentum," Physics Letters A, Vol. 370, No. 3-4, 2007, pp. 219-221; doi:10.1016/j.physleta.2007.05.079

[11] W. C. Qiang, R. S. Zhou and Y. Gao, "Any $\ell$-State Solutions of the Klein-Gordon with the Generalized Hulthén Potential," Physics Letters A, Vol. 371, No. 3, 2007, pp. 201-204; doi:10.1016/j.physleta.2007.04.109

[12] S. Dong, S.-H. Dong, H. Bahlouli and V. B. Bezzerra, "Algebraic Approach to the Klein-Gordon Equation with Hyperbolic Scarf Potential," International Journal of Modern Physics E, Vol. 20, No.1, 2011, pp. 55-61. doi:10.1142/S0218301311017326

[13] S. M. Ikhdair and J. Abu-Hasna, "Quantization Rule Solution to the Hulthén Potential in Arbitrary Dimension with a New Approximate Scheme for the Centrifugal Term," Physica Scripta, Vol. 83, No.2, 2011, pp. 025002-7. doi:10.1088/0031-8949/83/02/025002 
[14] G.-F. Wei, Z.-Z. Zhen and S.-H. Dong, "The Relativistic Bound and Scattering States of the Manning-Rosen Potential with an Improved New Approximate Scheme to the Centrifugal Term," Central European Journal of Physics, Vol. 7, No. 1, 2009, pp. 175-183. doi:10.2478/s11534-008-0143-9

[15] S.M. Ikhdair and R. Sever, "Approximate Bound State Solutions of Dirac Equation with Hulthén Potential Including Coulomb-Like Tensor Potential," Applied Mathematics and Computation, Vol. 216, No. 3, 2010, pp. 911923. doi:10.1016/j.amc.2010.01.104

[16] S. Flügge, "Practical Quantum Mechanics," Vol. 1, Springer, Berlin, 1994.

[17] J. Y. Guo, J. Meng and F. X. Xu, "Any $\ell$-State Solutions of the Klein-Gordon Equation with Special Hulthén Potentials," Chiniese Physics Letters, Vol. 20, 2003, pp. 602-604.

[18] A. D. Alhaidari, "Solution of the Relativistic Dirac-Hulthen Problem," Journal of Physics A, Vol. 37, 2004, pp. 5805-5813. doi:10.1088/0305-4470/37/22/007

[19] O. Bayrak, G. Kocak and I. Boztosun, "Any $\ell$-State Solutions of the Hulthén Potential by the Asymptotic Iteration Method," Journal of Physics A: Mathematical and General, Vol. 39, 2006, pp. 11521-11529. doi:10.1088/0305-4470/39/37/012

[20] S. Haouat and L. Chetouani, "Approximate Solutions of Klein-Gordon and Dirac Equations in the Presence of the Hulthén Potential," Physica Scripta, Vol. 77, 2008, pp. 025005-6; doi:10.1088/0031-8949/77/02/025005

[21] E. Olğar, R. Koç and H. Tütüncüler, "The Exact Solution of the $s$-Wave Klein-Gordon Equation for the Generalized Hulthén Potential by the Asymptotic Iteration Method," Physica Scripta, Vol. 78, 2008, pp. 015011-4;

[22] C. S. Jia, J. Y. Liu and P. Q. Wang, "A New Approximation Scheme for the Centrifugal Term and Hulthén Potential," Physics Letters A, Vol. 372, 2008, pp. 4779-4782.

[23] S.-H. Dong, "A New Quantization Rule to the Energy Spectra for Modified Hyperbolic-Type Potentials," International Journal of Quantum Chemistry, Vol. 109, No. 4, 2009, pp. 701-707. doi:10.1002/qua.21862

[24] M. F. Manning, "Exact Solutions of the Schrödinger Equation," Physical Review, Vol. 48, 1935, pp. 161-164. doi:10.1103/PhysRev.48.161

[25] A. Diaf, A. Chouchaoui and R. L. Lombard, "Feynman Integral Treatment of the Bargmann Potential," Annals of Physics, Vol. 317, 2005, pp. 354-365. doi:10.1016/i.aop.2004.11.010

[26] G.-F. Wei, C.-Y. Long and S.-H. Dong, "The Scattering of the Manning- Rosen Potential with Centrifugal Term," Physics Letters A, Vol. 372, No. 15, 2008, pp. 2592-2596. doi:10.1016/j.physleta.2007.12.042

[27] S.-H. Dong and J. Garcia-Ravelo, "Exact Solutions of the $s$-Wave Schrödinger Equation with Manning-Rosen Potential," Physica Scripta, Vol. 75, 2007, pp. 307-309. doi:10.1088/0031-8949/75/3/013

[28] W.-C. Qiang and S. H. Dong, “Analytical Approximation to the Solutions of the Manning-Rosen Potential with Centrifugal Term," Physics Letters A, Vol. 368, No.1-2, 2007, pp. 13-17. doi:10.1016/j.physleta.2007.03.057

[29] W.-C. Qiang and S.-H. Dong, "The Manning-Rosen Potential Studied by a New Approximate Scheme to the Centrifugal Term," Physica Scripta, Vol. 79, No. 4, 2009, p. 045004. doi:10.1088/0031-8949/79/04/045004

[30] C. Y. Chen, F. L. Lu and D. S. Sun, "Exact Solutions of Scattering States for the $s$-Wave Schrödinger Equation with the Manning-Rosen Potential," Physica Scripta, Vol. 76, 2007, pp. 428-430. doi:10.1088/0031-8949/76/5/003

[31] S. M. Ikhdair, "On the Bound-State Solutions of the Manning-Rosen Potential Including an Improved Approximation to the Orbital Centrifugal Term," Physica Scripta, Vol. 83, 2011, pp. 015010-10. doi:10.1088/0031-8949/83/01/015010

[32] E. Olğar, R. Koç and H. Tütüncüler, "Bound States of the $s$-Wave Equation with Equal Scalar and Vector Standard Eckart Potential," Chinese Physics Letters, Vol. 23, 2006, pp. 539-541. doi:10.1088/0256-307X/23/3/004

[33] W.-C. Qiang, J.-Y. Wu and S.-H. Dong, "The EckartLike Potential Studied by a New Approximate Scheme to the Centrifugal Term," Physica Scripta, Vol. 79, No. 6, 2009, p. 065011. doi:10.1088/0031-8949/79/06/065011

[34] X. Zou, L. Z. Yi and C. S. Jia, "Bound States of the Dirac Equation with Vector and Scalar Eckart Potentials," Physics Letters A, Vol. 346, 2005, pp. 54-64. doi:10.1016/j.physleta.2005.07.075

[35] G.-F. Wei, S.-H. Dong and V. B. Bezerra, "The Relativistic Bound and Scattering States of the Eckart Potential with a Proper New Approximate Scheme for the Centrifugal Term," International Journal of Modern Physics $A$, Vol. 24, No. 1, 2009, pp. 161-172. doi:10.1142/S0217751X09042621

[36] C. S. Jia, P. Guo and X. L. Peng, "Exact Solutions of the Dirac-Eckart Problem with Spin and Pseudospin Symmetry," Journal of Physics A: Mathematical and Theoretical, Vol. 39, No. 24, 2006, pp. 7737-7744. doi: 10.1088/0305-4470/39/24/010

[37] S. H. Dong, W. C. Qiang, G. H. Sun and V. B. Bezerra, "Analytical Approximations to the $\ell$-Wave Solutions of the Schrödinger Equation with the Eckart Potential," Journal of Physics A: Mathematical and Theoretical, Vol. 40, No. 34, 2007, pp. 10535-10540. doi: $10.1088 / 1751-8113 / 40 / 34 / 010$

[38] W.-C. Qiang and S.-H. Dong, “Analytical Approximation to the $\ell$-Wave Solutions of the Klein-Gordon Equation for a Second Pöschl-Teller Like Potential," Physics Letters A, Vol. 372, No. 27-28, 2008, pp. 4789-4792. doi:10.1016/j.physleta.2008.05.020

[39] S. M. Ikhdair, "Approximate Solutions of the Dirac Equation for the Rosen-Morse Potential Including the Spin-Orbit Centrifugal Term," Journal of Mathematical Physics, Vol. 51, No. 2, 2010, pp. 023525-16; doi:10.1063/1.3293759

[40] G.-F. Wei and S.-H. Dong, "Pseudospin Symmetry for Modified Rosen-Morse Potential Including a Pekeris- 
Type Approximation to the Pseudo-Centrifugal Term," The European Physical Journal A, Vol. 46, No. 2, 2010, pp. 207-212. doi:10.1140/epja/i2010-11031-0

[41] C. Eckart, "The Penetration of a Potential Barrier by Electrons," Physics Review, Vol. 35, No. 11, 1930, pp. 1303-1309. doi:10.1103/PhysRev.35.1303

[42] F. Cooper, A. Khare and U. Sukhatme, "Supersymmetry and Quantum Mechanics," Physics Report, Vol. 251, No. 5-6, 1995, pp. 267-385. doi:10.1016/0370-1573(94)00080-M

[43] J. J. Weiss, "Mechanism of Proton Transfer in Acid-Base Reactions," Journal of Chemical Physics, Vol. 41, 1964, pp. 1120-1124. doi:10.1063/1.1726015

[44] A. Cimas, M. Aschi, C. Barrientos, V. M. Rayőn, J. A. Sordo and A. Largo, "Computational Study on the Kinetics of the Reaction of $\mathrm{N}\left({ }^{4} \mathrm{~S}\right)$ with $\mathrm{CH}_{2} \mathrm{~F}$," Chemical Physics Letters, Vol. 374, No. 5-6, 2003, pp. 594-600. doi:10.1016/S0009-2614(03)00771-1

[45] C. S. Jia, X. L. Zeng and L. T. Sun, "PT Symmetry and Shape Invariance for a Potential Well with a Barrier," Physics Letters A, Vol. 294, No. 3-4, 2002, pp. 185-189. doi:10.1016/S0375-9601(01)00840-4

[46] C. S. Jia, Y. Li, Y. Sun, J. Y. Liu and L. T. Sun, "Bound States of the Five Parametric Exponential-Type Potential Model," Physics Letters A, Vol. 311, No. 2-3, 2003, pp. 115-125. doi:10.1016/S0375-9601(03)00502-4

[47] H. Eğrifes, D. Demirhan and F. Büyükkılıç, "Exact Solutions of the Schrödinger Equation for Two Deformed Hyperbolic Molecular Potentials," Physica Scripta, Vol. 60, No.3, 1999, pp. 195-198.

doi:10.1238/Physica.Regular.060a00195

[48] A. Arai, "Exactly Solvable Supersymmetric Quantum Mechanics," Journal of Mathematical Analysis and Applications, Vol. 158, No.1, 1991, pp. 63-79. doi:10.1016/0022-247X(91)90267-4

[49] R. Dutt, A. Khare and U. Sukhatme, "Supersymmetry, Shape Invariance, and Exactly Solvable Potentials," American Journal of Physics, Vol. 56, No.2, 1988, pp. 163-168. doi: $10.1119 / 1.15697$

[50] R. De, R. Dutt and U. Sukhatme, "Mapping of Shape Invariant Potentials Under Point Canonical Transformations," Journal of Physics A: Mathematical and General, Vol. 25, No. 13, 1992, pp. L843-L850. doi:10.1088/0305-4470/25/13/013

[51] M. Hruska, W.Y. Keung and U. Sukhatme, "Accuracy of semi classical Methods for Shape-Invariant Potentials," Physics Review A, Vol. 55, No. 5, 1997, pp. 3345-3350. doi:10.1103/PhysRevA.55.3345

[52] L.Z. Yi, Y.F. Diao, J.Y. Liu and C.S. Jia, "Bound States of the Klein-Gordon Equation with Vector and Scalar Rosen-Morse-Type Potentials," Physics Letters A, Vol. 333, 2004, pp. 212-217. doi:10.1016/j.physleta.2004.10.054

[53] R.L. Greene and C. Aldrich, "Variational Wave Functions for a Screened Coulomb Potential," Physics Review $A$, Vol. 14, 1976, pp. 2363-2366.
doi:10.1103/PhysRevA.14.2363

[54] G. F. Wei, C. Y. Long, X. Y. Duan and S. H. Dong, “Arbitrary $\ell$-Wave Scattering State Solutions of the Schrödinger Equation for the Eckart Potential," Physica Scripta, Vol. 77, 2008, pp. 035001-5. doi:10.1088/0031-8949/77/03/035001

[55] C. Y. Chen, D. S. Sun and F.L. Lu, "Analytical Approximations of Scattering States to the $\ell$-Wave Solutions for the Schrödinger Equation with the Eckart Potential," Journal of Physics A: Mathematical and Theoretical, Vol. 41, No. 3, 2008, pp. 035302. doi:10.1088/1751-8113/41/3/035302

[56] A. Soylu, O. Bayrak and I. Boztosun, “ $\kappa$-State Solutions of the Dirac Equation for the Eckart Potential with Pseudospin- and Spin-Symmetry," Journal of Physics A: Mathematical and Theoretical, Vol. 41, 2008, pp. 065308-8. doi:10.1088/1751-8113/41/6/065308

[57] L. H. Zhang, X. P. Li and C. S. Jia, "Analytical Approximation to the Solution of the Dirac Equation with the Eckart Potential Including the Spin-Orbit Coupling Term," Physics Letters A, Vol. 372, 2008, pp. 2201-2207. doi:10.1016/j.physleta.2007.11.022

[58] Y. Zhang, "Approximate Analytical Solutions of the Klein-Gordon Equation with scalar and Vector Eckart Potentials," Physica Scripta, Vol. 78, 2008, pp. 015006-4. doi:10.1088/0031-8949/78/01/015006

[59] C. S. Jia, J. Y. Liu and P. Q. Wang, "A New Approximation Scheme for the Centrifugal Term and the Hulthén Potential," Physics Letters A, Vol. 372, No. 27-28, 2008, pp. 4779-4782. doi:10.1016/j.physleta.2008.05.030

[60] A. F. Nikiforov and V. B. Uvarov, "Special Functions of Mathematical Physics,” Birkhauser, Bassel, 1988.

[61] S. M. Ikhdair and R. Sever, "Exact Quantization Rule to the Kratzer-Type Potentials: An Application to the Diatomic Molecules," Journal of Mathematical Chemistry, Vol. 45, No. 4, 2009, pp. 1137-1152. doi:10.1007/s10910-008-9438-8

[62] S. M. Ikhdair and R. Sever, "Solutions of the SpatiallyDependent Mass Dirac Equation with the Spin and Pseudospin Symmetry for the Coulomb-Like Potential," Applied Mathematics and Computation, Vol. 216, No. 2 , 2010, pp. 545-555. doi:10.1016/j.amc.2010.01.072

[63] S. M. Ikhdair, C. Berkdemir and R. Sever, "Spin and Pseudospin Symmetry Along With Orbital Dependency of the Dirac- Hulthén Problem," Applied Mathematics and Computation, Vol. 217, No. 22, 2011, pp. 9019-9032. doi:10.1016/j.amc.2011.03.109

[64] S. M. Ikhdair and R. Sever, "Bound-States of a SemiRelativistic Equation for the PT-Symmetric Generalized Potential by the Nikiforov-Uvarov Method," International Journal of Modern Physics E, Vol. 17, No. 6, 2008, pp. 1107-1123. doi:10.1142/S0218301308010337

[65] A. de Souza Dutra and G. Chen, "On Some Classes of Exactly-Solvable Klein-Gordon Equations," Physics Letters A, Vol. 349, 2006, pp. 297-301. doi:10.1016/j.physleta.2005.09.056 
[66] G. Chen, "Solutions of the Klein-Gordon for Exponential Scalar and Vector Potentials," Physics Letters A, Vol. 339, 2005, pp. 300-303. doi:10.1016/i.physleta.2005.03.040

[67] S. M. Ikhdair, "Rotation and Vibration of Diatomic Molecule in the Spatially-Dependent Mass Schrodinger Equation with Generalized $q$-Deformed Morse Potential," Chemical Physics, Vol. 361, No. 1-2, 2009, pp. 9-17. doi:10.1016/j.chemphys.2009.04.023

[68] N. Saad, "The Klein-Gordon Equation with a Generalized Hulthén Potential in D-Dimensions," Physica Scripta, Vol. 76, 2007, pp. 623-627. doi:10.1088/0031-8949/76/6/005

[69] W. C. Qiang, R. S. Zhou and Y. Gao, "Any $\ell$-State Solutions of the Klein-Gordon Equation with the Generalized Hulthén Potential," Physics Letters A, Vol. 371, No. 3, 2007, pp. 201-204. doi:10.1016/j.physleta.2007.04.109

[70] S. M. Ikhdair and R. Sever, "Any $\ell$-State Improved Quasi-Exact Analytical Solutions of the Spatially Dependent Mass Klein-Gordon Equation for the Scalar and Vector Hulthén Potentials," Physica Scripta, Vol. 79, No. 3, 2009, pp. 035002-12. doi:10.1088/0031-8949/79/03/035002

[71] N. Rosen and P. M. Morse, "On the Vibrations of Polyatomic Molecules,” Physics Review, Vol. 42, No. 2, 1932, pp. 210-217. doi:10.1103/PhysRev.42.210

[72] A. S. de Castro, "Klein-Gordon Particles in Mixed Vector-Scalar Inversely Linear Potentials," Physics Letters A, Vol. 338, 2005, p. 81. doi:10.1016/j.physleta.2005.02.027

[73] F. Cooper, A. Khare and U. P. Sukhatme, "Supersymmetry in Quantum Mechanics," World Scientific, Singapore, 2001.

[74] C. M. Bender and S. Boettcher, "Real Spectra in NonHermitian Hamiltonians Having PT Symmetry," Physical Review Letters, Vol. 80, No. 24, 1998, pp. 5243-5246. doi:10.1103/PhysRevLett.80.5243

[75] C. B. C. Jasso, "Baryon Spectra in a Quark-Diquark Model with the Trigonometric Rosen- Morse Potential," MS Thesis (in Spanish), Institute of Physics, Autonomous University of San Luis Potosi, México, 2005.

[76] M. R. Spiegel, "Mathematical Handbook of Formulas and Tables," McGraw-Hill Publishing Company, New York, 1968.

[77] E. Eichten, K. Gottfried, T. Kinoshita, K.D. Lane and T.M. Yan, "Charmonium: Comparison with Experiment," Physical Review D, Vol. 21, No. 1, 1980, pp. 203-233. doi:10.1103/PhysRevD.21.203 